\title{
Agreement Between Retrospective Accounts of Substance Use and Earlier Reported Substance Use
}

\author{
Linda M. Collims, dohn W. Graharm, William B. Hansen, \\ and G. Anderson Johnson

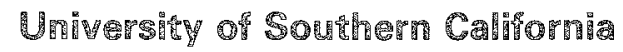

This present study examined agreement between retrospective accounts of substance use and earlier reported substance use in a high school age sample. Three issues were addressed: (1) extent of overall agreement; (2) evidence for the presence of a response-shift bias; and (3) extent to which current use biases recall of substance use. Subjects were 415 high school students who took part in a smoking prevention program. At the last measurement, which took place $21 / 2$ years after the pretest, the students were asked to recall pretest use of tobacco, alcohol, and marijuana, and use one year earlier. Results showed an overall tendency for students to recall less use of uncontrolled substances than had been previously reported. For the one controlled substance included in the questionnaire, marijuana, current nonusers tended to recall less use than they had reported at the time, whereas current users tended to recall more use than had been reported. The present study found no evidence for a response-shift bias. It is suggested that the explicitly worded anchors on the response scales helped prevent such a bias. Finally, the results suggest that current use biases recall of past use to a substantial extent, and that this bias affects recall of alcohol use most severely.

Retrospective reports of behavior are used mainly under two sets of circumstances. First, retrospective reports may be used as a substitute when concurrent data are not available. For example, some-

APPLIED PSYCHOLOGICAL MEASUREMENT

Vol. 9, No. 3, September 1985, pp. 301-309

(C) Copyright 1985 Applied Psychological Measurement Inc. 0146-6276/85/030301-09\$1.70 times crucially relevant phenomena, for example, the drinking behavior of an alcoholic being admitted to treatment, have taken place before the beginning of a study or treatment program. Another instance when retrospective reports could be used as a substitute for concurrent data is in panel studies. If a subject is absent for an intermediate wave of data collection, a retrospective account collected at a subsequent wave could be inserted to replace the missing observation (Finney, 1981).

The second set of circumstances under which retrospective reports of behavior are used is when a response-shift bias may be operating (Howard, Millham, Slaten, \& O'Donnell, 1981; Howard, Ralph, Gulanick, Maxwell, Nance, \& Gerber, 1979). The response-shift bias affects pretest-posttest designs in which a treatment group receives an intervention intended to alter behavior. When a behavioral intervention alters not only the treatment subjects' behavior, but also their awareness of the behavior and their judgment about their own level of skill, the treatment subjects may undergo a change in their interpretation of the anchors of a response scale. This change is what is meant by responseshift bias. Howard et al. (1979) suggested that when a response-shift bias is operating, retrospective reports can be used to obtain a more nearly accurate assessment of pretest-posttest change.

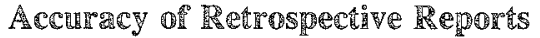

Although relatively little research has been done on the advisability of substituting retrospective re- 
ports for concurrent reports, there is some evidence that retrospective reports can be adequate indicators of past behavior. Maisto, Sobell, Cooper, and Sobell (1982) compared two interview techniques for obtaining retrospective reports of drinking-related behavior from alcohol abusers. Examination of individual items included in their study reveals that more specific questions, such as those asking how many days the subject spent in jail for alcoholrelated offenses in the last 30 days, tended to show a higher degree of agreement. In an investigation of agreement between recalled data and past reported data, Finney (1981) addled a set of questions to the second wave of a panel study. These new questions asked for recall on several items included in the first wave of measurement four years earlier. For example, one question was, "Back in 1967 . would you say your own health in general was excellent, good, fair, or poor?' Finney found that subjective questions asking for evaluations, such as the health question above, showed less agreement between first-wave response and recall than did more objective questions, such as whether or not the subject grew his or her own food. Taken together, the Finney (1981) and Maisto et al. (1982) studies suggest that moderate to high recall accuracy is possible when retrospective questions are relatively specific and objective.

Another study, however, found less encouraging results. Simpura and Poikolainen (1983) asked Finnish men to recall their alcohol use 18 years ago, and compared the recall with alcohol consumption reported in an interview occurring 18 years previously. They found that the recalled use tended to be considerably higher than the subjects' reported use at the first interview, and overall agreement between retrospective and concurrent reports was quite low. Of course, the original report and later recall in this study were separated by 18 years, which may be an unreasonably long time over which to expect subjects to remember. Simpura and Poikolainen considered the possibility that the drastic increase in per capita consumption of alcohol in Finland since the first interview may have affected the respondents' perceptions of their past habits. However, they found in regression analyses that current use did not predict recalled use. Although no evidence for bias attributable to current use was found in this study, the possibility that current use influences recall of use should not be discarded. Simpura and Poikolainen's $N$ was only 67, which allowed only a weak test of the hypothesis that current use affects recall of past use.

\section{The Present Stady}

The present study addressed several interesting questions raised by this literature. The first question was: To what extent does recall of past substance use agree with past concurrent reports of substance use? In the case of alcohol, the Maisto et al. (1982) study leads to the expectation of a great deal of agreement, whereas Simpura and Poikolainen (1983) suggested that there may be relatively little agreement. The present study examined the correspondence between past reported use and recalled use for tobacco and marijuana as well as for alcohol.

In the present study, subjects took part in a smoking prevention program for high school students (Johnson, Hansen, Collins, \& Graham, 1984). Thus, the second question addressed by the present study was: Will a smoking prevention program produce a response-shift bias? It is possible that an increased awareness of smoking behavior due to the intervention could cause a response-shift bias in reported tobacco use. Because some diffusion of treatment effects over alcohol and marijuana were found (Johnson et al., 1984), reported use of these substances was examined for response-shift bias as well.

A third question addressed here was: Does current substance use bias recall of past substance use? Simpura and Poikolainen (1983) raised this interesting possibility, and though they concluded that there were no biasing effects of current use in their study, the larger $N$ of the present study allows a more powerful test of the hypothesis.

\section{Method}

Subjects were high school students in the Los Angeles area who were participants in the High School Anti-Smoking Project (HASP). As part of this project, students completed a questionnaire in 
January 1981 and May 1982. The questionnaire contained items asking about tobacco, alcohol, and marijuana use. Three questions $(1,2$, and 3$)$ asked about use habits, and four questions $(4,5,6$, and 7) asked if subjects had ever tried a substance. The questions and response categories are shown in $\mathrm{Ta}$ ble 1 .

In the spring of 1983 the questionnaire was administered again, with the addition of a supplemental questionnaire. The supplemental questionnaire asked subjects to recall their tobacco, alcohol, and marijuana use habits one year earlier, that is, at the time of the May 1982 questionnaire, and $21 / 2$ years earlier, that is, at the time of the January 1981 questionnaire. Each substance use question and set of response categories was worded as simillarly as possible to its earlier counterpart, so as to maximize the possibility of exact recall. The rewording of the questions also appears in Table 1. A total of 415 students who were present at the January 1981 data collection and the May 1982 data collection also completed the supplemental questionnaire. However, due to missing data on individual items, the $N$ varied slightly among analyses.

\section{Resule}

Overar Agreement Between

Past Repored Use atid Mecalled Use

Overall agreement between past reported use and recalled use was examined in three ways: (1) percent exact agreement between reported use and recalled use; (2) a measure of association ( $r$ ) for the use habits items, and Cohen's kappa (1968) for the dichotomous "ever tried" items; and (3) $t$-tests of the mean differences between reported use and recalled use. These results are presented in Table 2 for the use habits items and Table 3 for the "ever tried" items. For both the use habits items and the "ever tried" items, marijuana showed the greatest degree of agreement, while both the alcohol use habits and "ever tried" items showed the lowest degree of agreement.

Differences between mean reported use and mean recalled use were significant in every case except for the marijuana use habits item involving use $21 / 2$ years ago. In every case, recalled use is less than was reported at the time, with one exception: Interestingly, subjects recalled more marijuana use than they had reported one year earlier.

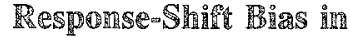 Reported Sulosidnce Use}

In a pretest-postest design, presumably there are no between-group differences at pretest in interpretation of the dependent measure response scales. If the treatment produces a response-shift bias, then at posttest the treatment subjects use the response scale differently from control subjects. When asked to recall pretest behavior, treatment subjects are now using a response scale different from the one on which they based their original pretest report. It follows from this that if a response-shift bias occurs, then the mean difference between pretest and recalled pretest should vary by condition. Accordingly, $t$-tests were used to test for differences between the treatment and control groups on the disparity between reported pretest use habits and later recalled use habits. No significant differences were found for tobacco, alcohol, or marijuana use habits (all $p s>.15$ ).

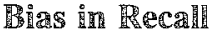 \\ Dute 10 Cringent Use}

If both the reported past use and recall measures used in this study were perfectly valid and reliable, then the correlation between them would be 1.0 . In the present study this correlation is less than perfect, as Table 2 shows, meaning that some of the variance in recall is left unaccounted for by reported past use. This leftover variance may not be all random error. If current use is a significant biasing factor in recalled use, then current use should account for some of this leftover variance; that is, current use should reliably predict the variance in recalled use that is left after the effects of reported past use have been partialled out.

The model of the present study is the following:

$$
\text { Recalled Use }=\text { Past Use + Current Use }
$$

Any variance in recalled use that is attributable to 


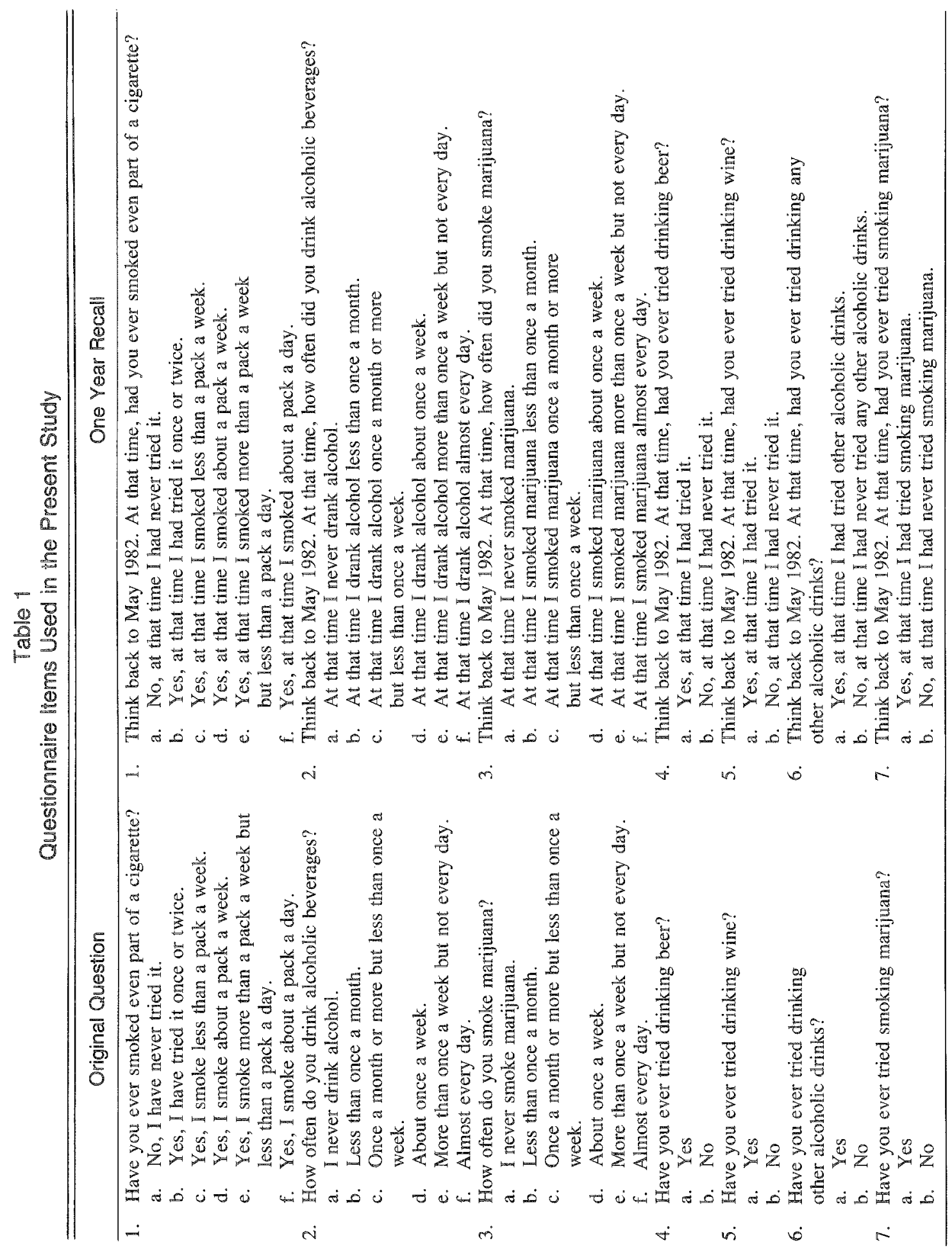


Table 2

Agreement Between Past Reported Substance Use Habits

and Recalled Use Habits

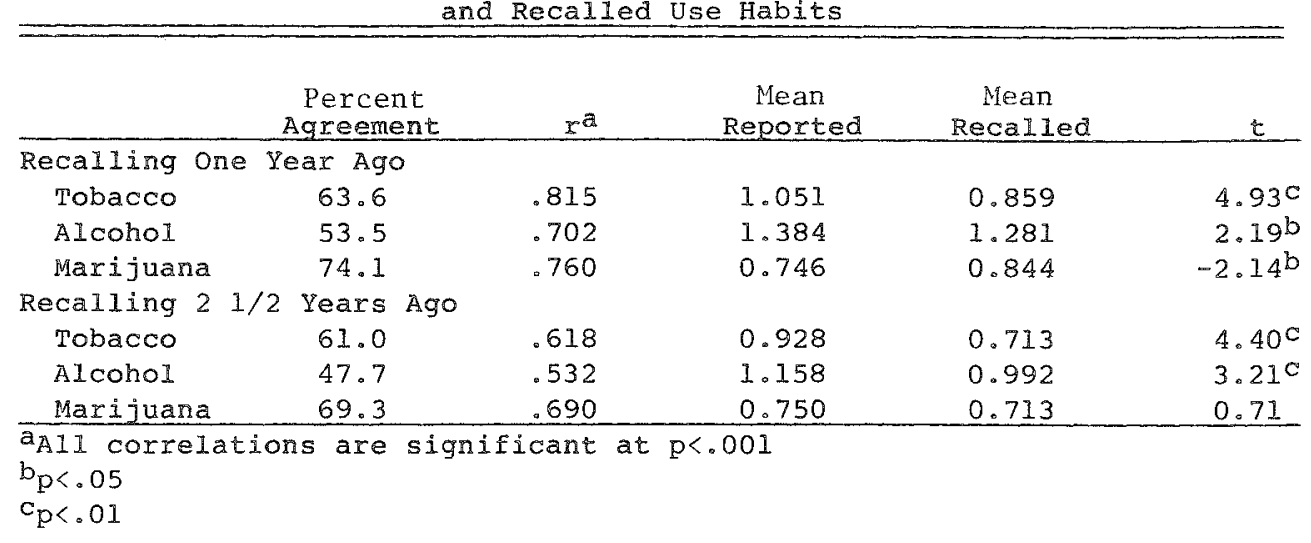

past use, including the part of past use that is correlated with current use, is considered legitimate. Any additional independent contribution of current use is considered bias.

Accordingly, a series of hierarchical regressions predicting recalled use was done, with past use always entered into the model before current use. This order of inclusion allowed all of past use, including the part that overlaps with current use, to account for variance in recalled use.

The results appear in Table 4. In these analyses past use always accounts for more of the variance than current use does, as might be expected. However, current use explains a significant amount of additional variance in every case. For one-year recall, the contribution of current alcohol use is strongest, both in absolute and in relative terms, when compared to the contribution of past use. For $2 \frac{1}{2}$-year recall, the contribution of current tobacco use is strongest in absolute terms, but current alcohol use accounts for relatively more of the variance in recalled alcohol use when compared to the contribution of past use. For all three substances, the variance attributable to current use increases from the one-year interval to the $2 \frac{1}{2}-2$-year interval, both in terms of absolute $R^{2}$ and in terms of the relative contribution to overall $R^{2}$.

The analyses shown in Table 4 include all stu.

Table 3

Agreement Between Past Reported Substance Trying and Recalled Substance Trying

\begin{tabular}{|c|c|c|c|c|c|}
\hline Substance & $\begin{array}{c}\text { Percent } \\
\text { Agreement }\end{array}$ & $K^{*}$ & $\begin{array}{l}\text { Percent } \\
\text { Reported }\end{array}$ & $\begin{array}{l}\text { Percent } \\
\text { Recalled }\end{array}$ & $t^{*}$ \\
\hline \multicolumn{6}{|c|}{ Recalling One Year Ago } \\
\hline Tobacco & 77.4 & .553 & 66.7 & 48.7 & 8.05 \\
\hline Beer & 85.6 & .539 & 85.1 & 76.6 & 4.57 \\
\hline Wine & 87.6 & .559 & 87.8 & 78.9 & 5.26 \\
\hline Liquor & 82.3 & .529 & 79.2 & 71.3 & 3.80 \\
\hline Marijuana & 89.1 & .782 & 52.5 & 46.0 & 3.99 \\
\hline \multicolumn{6}{|c|}{ Recalling $21 / 2$ Years Ago } \\
\hline Tobacco & 75.6 & .522 & 61.5 & 45.9 & 6.59 \\
\hline Beer & 79.4 & .460 & 81.4 & 68.8 & 5.74 \\
\hline Wine & 80.7 & .439 & 82.4 & 74.0 & 3.92 \\
\hline Liquor & 73.6 & .403 & 73.1 & 62.3 & 4.16 \\
\hline Marijuana & 82.7 & .640 & 42.6 & 36.8 & 2.79 \\
\hline
\end{tabular}

Downloaded from the Digital Conservancy at the University of Minnesota, http://purl.umn.edu/93227. May be reproduced with no cost by students and faculty for academic use. Non-academic reproduction requires payment of royalties through the Copyright Clearance Center, http://www.copyright.com/ 


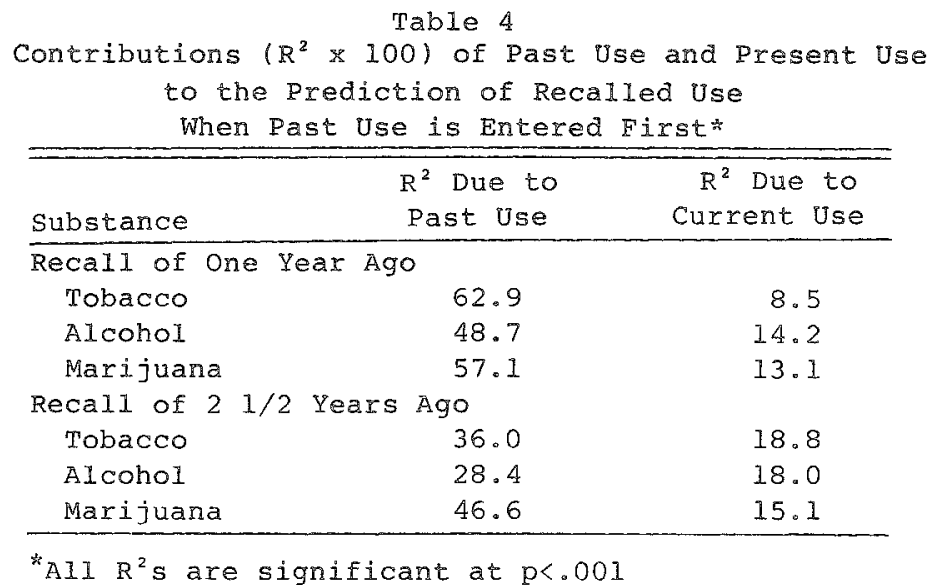

dents. However, for many of these students substance use did not change from the past to the present, as measured concurrently at each wave. For these students, the correlation between past use and current use is perfect, so after past use is entered into a regression equation predicting recalled wse, current use adds nothing. For students whose current use is different from past use, the contribution of current use to recalled use will probably be larger than that shown in Table 4. Therefore, the regression analyses shown in Table 4 were redone including only students whose use habits changed. These results are shown in Table 5 .

For this subset of students, the contribution of current use is larger than it is for the group as a whole, ranging from $19 \%$ to $25 \%$ of the variance at the one-year interval and from $23 \%$ to $28 \%$ of the variance at the $2 \frac{1}{2}-y$ year interval. The $R^{2}$ attributable to past use is lower overall for the students whose use habits changed. Based on these students, only the one-year recall of tobacco use appears to be relatively uncontaminated by current use. All other recall measures are considerably biased, particularly the 21/2-year recall of alcohol use habits, in which the contribution of current use was twice that of past use.

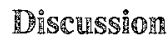

Overall ACCunracy of petrospective Reports

The present study showed that there was an over- all tendency for students to recall less use than originally reported for the two uncontrolled substances, tobacco and alcohol. One possible explanation for this is that young students prefer to think of their substance use as a relatively recent development rather than a deeply ingrained habit. Thus, they may tend to deny or minimize past levels of use. Another possible explanation is that as time elapses and memory fades, students may fail to remember substance use occasions, and simply think their use was less because it seems so in retrospect.

For marijuana, however, students recalled more use than was reported one year ago, with no difference between recalled use and reported use $21 / 2$ years earlier. One possible explanation for this lies in an important difference between alcohol and tobacco on the one hand and marijuana on the other. Because marijuana is a controlled substance, it may be that past levels of use were underreported due to fears that the experimenters would single out students and report them to the authorities. By the last of four waves of measurement, which is when the recall instrument was administered, the students can be more confident that this will not happen. In addition, it is even less likely that reports of use a year or two ago will cause trouble for them. Thus, the students who were actually using marijuana one year or $2 \frac{1}{2}$ years ago may be likely to recall using more marijuana than they reported at the time. Because the past reported marijuana use is likely to be incorrect, this hypothesis cannot be tested by dividing the students into past users and past non- 


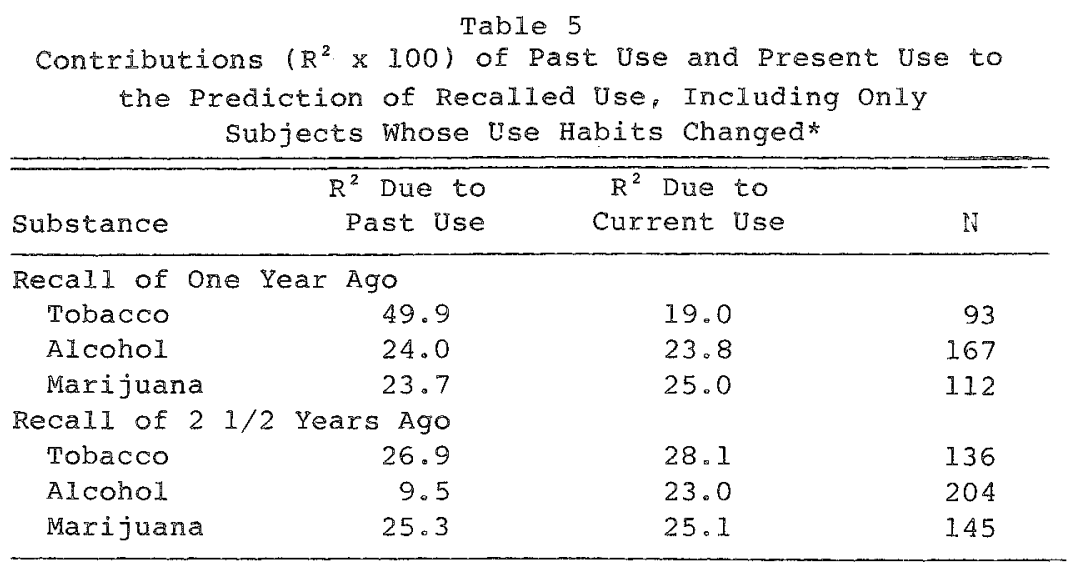

${ }^{*} \mathrm{~A} 11 \mathrm{R}^{2} \mathrm{~s}$ are significant at $\mathrm{p}<.01$

users; however, this can be approximated by dividing the group into current users and current nonusers.

This division was done and an analysis of variance was performed with one between-subjects factor, current use, and two within-subjects factors, type of measure (report or recall) and time (one year ago or $2 \frac{1}{2}$ years ago). The analysis found a significant current use by type of measure interaction $(R[1,378]=12.21, p<.001)$ consistent with the hypothesis that marijuana users tend to recall more use than originally reported, whereas nonusers do not show this tendency. In fact, there was no difference between reported use and recalled use of one year ago for nonusers; however, for $2 \frac{1 / 2}{2}$ year recall, current nonusers showed the same tendency to recall less use than previously reported that was in evidence for tobacco and alcohol.

Thus for a controlled substance, marijuana, current nonusers recalled less use than previously reported, whereas current users recalled more use than previously reported. These currently-using students may still be recalling less marijuana use than they actually experienced, but more than they felt comfortable reporting earlier. Another possibility is that marijuana users may exaggerate past use because they feel that marijuana use is a desirable or high-status activity. However, this does not explain why such tendencies would exist for marijuana and not for alcohol or tobacco use.

\section{Response-Shini: 呫ids}

The present study found no evidence for a response-shift bias in these data. A likely reason for the null results is the type of response scale anchors used. The present study used very explicit anchors, expressing substance use behavior in terms of daily, weekly, and monthly frequency. The work of Howard and colleagues (Howard et al., 1979; Howard et al., 1981) generally used less explicit anchors requiring subjective judgments, such as "average" and "good." Although the present study did not provide a direct comparison of explicit versus nonexplicit response-scale anchors, it is predicted that such a study would find a much smaller response-shift bias effect if explicit anchors were used. This conjecture is supported by Maisto et al. (1982) and Finney (1981), who found better recall accuracy using explicit questions.

\section{Curiorent Substance Use Bias of Recalled Suhbstance Use}

Finally, the data showed some interesting results bearing on the issue of possible bias of recalled substance use by current substance use. When all students were considered, the bias was significant, but relatively small, ranging from about $12 \%$ to $22.6 \%$ of the total $R^{2}$ at the one-year interval, and from $25 \%$ to $29 \%$ of the total $R^{2}$ at the $21 / 2$-year interval. However, calculations based only on stu- 
dents whose use changed from wave to wave showed that $28 \%$ to $51 \%$ of the total $R^{2}$ was attributable to current use for one-year recall, and from $50 \%$ to $71 \%$ of the total $R^{2}$ was attributable to current use for $2 \frac{1}{2}$-year recall. These data also suggest that the current use bias operates in alcohol use recall to a particularly high degree. These results make the somewhat ironic suggestion that retrospective reports of use habits are most valid when use habits have not undergone change since the time period being recalled.

\section{Thmplications}

The results of the present study have methodological implications in three areas. First, these results suggest that experimenters should use caution in basing use rates of uncontrolled substances on retrospective self-reports. These rates are likely to be lower than would be obtained with concurrent reports. However, comparisons made between groups are likely to be valid, as long as the absolute rates are not interpreted. For controlled substances, retrospective reports may actually be more valid than a concurrent report, if the subject has any reason to fear legal ramifications from his or her answers. Perhaps some future study will speak to this issue more directly. For instance, it is possible that "bogus pipeline" techniques may be useful in encouraging more accurate concurrent reporting of marijuana use as has been suggested for tobacco self-reports (Evans, Hansen, \& Mittelmark, 1977; Luepker, Pechacek, Murray, Johnson, Hurd, \& Jacobs, 1981).

Second, the present study suggests that the use of objective, explicit anchors on response scales may help reduce or eliminate response-shift bias. Of course, such anchors do not make sense under all circumstances. Perhaps a future study will provide a direct comparison of response-shift bias produced with explicit versus nonexplicit anchors.

Finally, the results showed that retrospective accounts of past use habits are likely to be biased to a substantial degree by current use habits, especially if use habits have undergone change. The recall of alcohol use habits seems to be especially susceptible to this bias. The present results imply that in an adult population whose use habits have stabilized, retrospective reports may be quite valid. However, in a school-age or even college-age population whose use habits are likely to be changing rapidly, retrospective reports may be relatively invalid. Since alcohol use habits probably vary over time in any population more than most other substance use habits, alcohol use recall may be of extremely limited research utility.

\section{Refierences}

Cohen, J. (1968). Nominal scale agreement with provision for disagreement or partial credit. Psychological Bulletin, 70, 213-220.

Evans, R. I., Hansen, W. B., \& Mittelmark, M. B. (1977). Increasing the validity of self-reports of behavior in a smoking-in-children investigation. Joumal of Applied Psychology, 62, 521-523.

Finney, H. C. (1981). Improving the reliability of retrospective survey measures: Results of a longitudinal field survey. Evaluation Review, 5, 207-229.

Howard, G. S., Millham, J., Slaten, S., \& O'Donnell, L. (1981). Influence of subject response style effects on retrospective measures. Applied Psychological Measurement, 5, 89-100.

Howard, G. S., Ralph, K. M., Gulanick, N. A., Maxwell, S. E., Nance, D. W., \& Gerber, S. K. (1979). Internal invalidity in pretest-posttest self-report evaluations and a re-cvaluation of retrospective pretests. Applied Psychological Measurement, 3, 1-23.

Johnson, C. A., Hansen, W. B., Collins, L. M., \& Graham, J. W. (1984). High school smoking prevention: Results of a three-year longitudinal siudy. Manuscript submitted for publication.

Luepker, R. V., Pechacek, T. F., Murray, D. M., Johnson, C. A., Hurd, F., \& Jacobs, D. R. (1981). Saliva thiocyanate: A chemical indicator of smoking in adolescents. American Journal of Public Health, 71, 1320-1324.

Maisto, S. A., Sobell, L. C., Cooper, A. M., \& Sobell, M. B. (1982). Comparison of two techniques to obtain retrospective reports of drinking behavior from alcohol abusers. Addictive Behaviors, 7, 33-38.

Simpura, J., \& Poikolainen, K. (1983). Accuracy of retrospective measurement of individual alcohol consumption in men: A reinterview after 18 years. Journal of Studies on Alcohol, 12, 911-916.

\section{Acknowledgments}

The authors thank Nata Preis for conducting the data collection, and the Los Angeles Unified School District 
for their cooperation in making students available. This research was supported by Grant 1-R01-DA02941 from the National Institute on Drug Abuse, and a grant from the American Heart Association (Brian R. Flay, P.I.).

\section{Author's Address}

Send requests for reprints or further information to Linda M. Collins, Health Behavior Research Institute, University of Southern California Health Sciences Campus, 1985 Zonal Ave., Los Angeles CA 90033 U.S.A.

Downloaded from the Digital Conservancy at the University of Minnesota, http://purl.umn.edu/93227. May be reproduced with no cost by students and faculty for academic use. Non-academic reproduction requires payment of royalties through the Copyright Clearance Center, http://www.copyright.com/ 